\title{
Effectiveness of Ultrasound in Evaluation of Fatty Infiltration in Rotator Cuff Muscles
}

\author{
Bong-Kyung Park, MD, Seok-Ha Hong, MD, Woong-Kyo Jeong, MD \\ Department of Orthopedic Surgery, Korea University College of Medicine, Seoul, Korea
}

\begin{abstract}
Background: This study aimed to verify the diagnostic performance of Ultrasonography (US) in the evaluation of fatty infiltration (FI) in rotator cuff muscles and to analyze the diagnostic values of each measurement component.

Methods: The degree of FI in 108 shoulders was assessed by magnetic resonance imaging (MRI) and US. MRI findings were graded by the Goutallier classification. US findings were graded by the Strobel method. Agreement between MRI and US findings was evaluated. The sensitivity and specificity for detecting $\mathrm{Fl}$ and intraobserver reliabilities were also assessed.

Results: US grading of the infraspinatus based on short-axis architecture showed good agreement ( $\mathrm{k}=0.62)$. US grading-based on architecture showed good agreement for both supraspinatus and infraspinatus in long- and short-axis scans (supraspinatus, $\mathrm{K}=0.63$; infraspinatus, $\mathrm{K}=0.68$ ), while that based on echogenicity showed moderate agreement (supraspinatus, $\mathrm{K}=0.51$; infraspinatus, $\mathrm{K}=$ $0.50)$. The sensitivity, specificity, positive predictive value, negative predictive value, and accuracy of detecting advanced Fl were significant in both axes.

Conclusions: US is effective for detecting advanced FI in the rotator cuff muscles. US can assess the infraspinatus more accurately than the supraspinatus, and architecture is a more decisive component of Fl status than echogenicity.

Keywords: Shoulder, Rotator cuff muscle, Fatty infiltration, Ultrasonography, Infraspinatus, Architecture
\end{abstract}

Fatty infiltration (FI) in the muscles of the rotator cuff is associated with the negative prognosis of surgical repair of the rotator cuff, ${ }^{1-4)}$ and it can be used to predict the reparability of massive rotator cuff tears ${ }^{5}$ because the rate of FI is higher in patients with retears of rotator cuff muscles. ${ }^{3)}$ Therefore, preoperative evaluation of FI in the rotator cuff muscles is an important aspect of the treatment of rotator cuff tears.

Goutallier et al. ${ }^{2)}$ classified FI in the rotator cuff muscles into five stages based on preoperative computed tomography (CT) findings, and FI in the infraspinatus muscles was shown to have a highly negative effect on the surgical repair of the supraspinatus. Fuchs et al. ${ }^{6}$ graded

Received May 23, 2019; Accepted June 10, 2019

Correspondence to: Woong-Kyo Jeong, MD

Department of Orthopedic Surgery, Korea University College of Medicine,

73 Inchon-ro, Seongbuk-gu, Seoul 02841, Korea

Tel: +82-2-920-5924, Fax: +82-2-924-2471

E-mail: drshoulder@gmail.com
FI in the rotator cuff muscles on the basis of para-sagittal scans of magnetic resonance imaging (MRI), ${ }^{7)}$ which is widely accepted as the standard imaging modality for evaluating the degree of FI in these muscles.

Ultrasonography (US) has been used to diagnose rotator cuff tears for many years, ${ }^{8-10)}$ and the overall accuracy of US is comparable to that of MRI in the detection of rotator cuff tears. ${ }^{9)}$ It can also be used to evaluate FI in the rotator cuff muscles. ${ }^{11,12)}$ Wall et al. ${ }^{4)}$ reported that the diagnostic performance of US in identifying and grading FI in the rotator cuff muscles was comparable to that of MRI. The muscle architecture and echogenicity on US were graded by using three-point and dichotomous grading scales and compared with the FI grade on MRI. However, in their article, the authors did not provide methods for a detailed interpretation of various combined echogenicity and architecture findings. For example, in the case of grade II echogenicity and grade I architecture in the short-axis scan and different grades in the long-axis scan, the determination of the final FI grade is highly ambiguous, which 
Park et al. Ultrasonographic Evaluation of Rotator Cuff Muscle

Clinics in Orthopedic Surgery • Vol. 12, No. 1, $2020 \bullet$ www.ecios.org

makes the evaluation difficult to apply directly to patients. The aims of this study were to verify the diagnostic performance of US in evaluating FI in the rotator cuff muscles by using previously proposed methods and to analyze the diagnostic values of each component and scanning plane.

\section{METHODS}

\section{Study Subjects}

We conducted this study in compliance with the principles of the Declaration of Helsinki. The protocol of this study was reviewed and approved by the Institutional Review Board of Korea University Anam Hospital (IRB No. 2020AN0025). Written informed consents were obtained. The records of 151 patients who visited the shoulder and elbow clinics of our hospital from September 2015 to November 2016 were retrospectively evaluated. Patients were included if they had shoulder pain and underwent both MRI and US scans of the shoulder within 2 months. Patients were excluded if they underwent MRI evaluation in other clinics, owing to improper quality of images for evaluating the FI status. According to the above criteria, of 151 patients, 105 patients (108 shoulders) were included in this study. Demographic information, including sex and age, and information on the status of the rotator cuff were recorded.

The mean patient age at the time of US was 59 years (range, 19 to 86 years). Final diagnoses were made based on intraoperative findings. Unilateral rotator cuff tears were diagnosed in 100 patients and bilateral rotator cuff tears, in three patients, among a total of 108 shoulders with rotator cuff tears. Two patients had calcific tendinitis. We performed arthroscopic rotator cuff repair in 95 patients with rotator cuff tears (three were bilateral cases). Reverse total shoulder arthroplasty was performed in three patients with massive rotator cuff tears, arthroscopic debridement in two patients with calcific tendinitis, open rotator cuff repair in one patient with massive rotator cuff tear, and conservative management in the other patients with rotator cuff tears.

\section{Imaging Protocol}

MRI examinations were performed by using a standardized protocol for the evaluation of rotator cuff pathology. The standard MRI sequences included coronal turbo spinecho T1, coronal T2 with fat suppression, oblique sagittal turbo spin-echo T1, oblique sagittal T2 with fat suppres-

\section{Table 1. Three-Point Ultrasound Grading Scale for Fatty Infiltration in the Rotator Cuff Muscles

\begin{tabular}{ccc} 
Grade & \multicolumn{1}{c}{ Echogenicity } & \multicolumn{1}{c}{ Architecture } \\
\hline 0 & $\begin{array}{c}\text { Isoechoic to the overlying } \\
\text { muscle }\end{array}$ & $\begin{array}{c}\text { Clearly visible intramuscular } \\
\text { tendons and identifiable } \\
\text { pennate pattern }\end{array}$ \\
\hline 1 & $\begin{array}{c}\text { Slightly increased echogenicity } \\
\text { compared with the overlying } \\
\text { muscle }\end{array}$ & $\begin{array}{c}\text { Partially visible intramuscular } \\
\text { tendons and pennate pattern }\end{array}$ \\
\hline 2 & $\begin{array}{c}\text { Markedly increased } \\
\text { echogenicity compared with } \\
\text { the overlying muscle }\end{array}$ & $\begin{array}{c}\text { No discernible intramuscular } \\
\text { tendons or pennate pattern }\end{array}$ \\
\hline
\end{tabular}

Modified from Strobel et al. ${ }^{13)}$
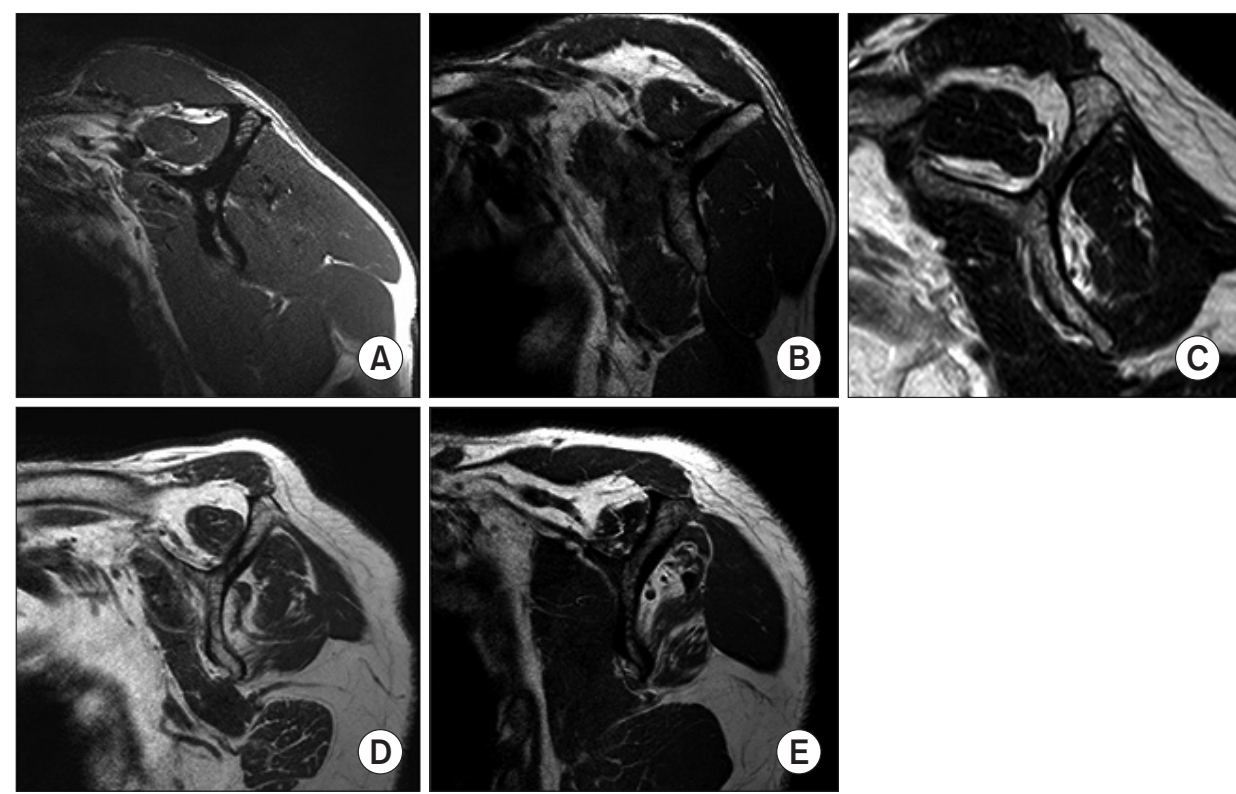

Fig. 1. Fuch's modification of the Goutallier magnetic resonance imaging (MRI) classification system. (A) T1-weighted MRI showing Goutallier grade 0 fatty infiltration (no fatty deposits) of the infraspinatus muscle. (B) T1-weighted MRI showing Goutallier grade 1 fatty degeneration (some fatty streaks) of the infraspinatus muscle. (C) T1-weighted MRI showing Goutallier grade 2 fatty degeneration (less fat than muscle) of the supraspinatus muscle. (D) T1-weighted MRI showing Goutallier grade 3 fatty degeneration (as much fat as muscle) of the infraspinatus muscle. (E) T1-weighted MRI showing Goutallier grade 4 fatty degeneration (more fat than muscle) of the infraspinatus muscle. 
Park et al. Ultrasonographic Evaluation of Rotator Cuff Muscle

Clinics in Orthopedic Surgery • Vol. 12, No. 1, $2020 \bullet$ www.ecios.org

sion, and axial proton density-weighted images with fat suppression. The oblique sagittal views were extended at least $1 \mathrm{~cm}$ medial to the spinoglenoid notch in order to include sections showing the muscle bulks in the supraspi- natus and infraspinatus fossae.

The images were graded in a blinded fashion by one orthopedic specialist (WKJ) in our institution. A single T1-weighted oblique sagittal section within $1 \mathrm{~cm}$ medial to
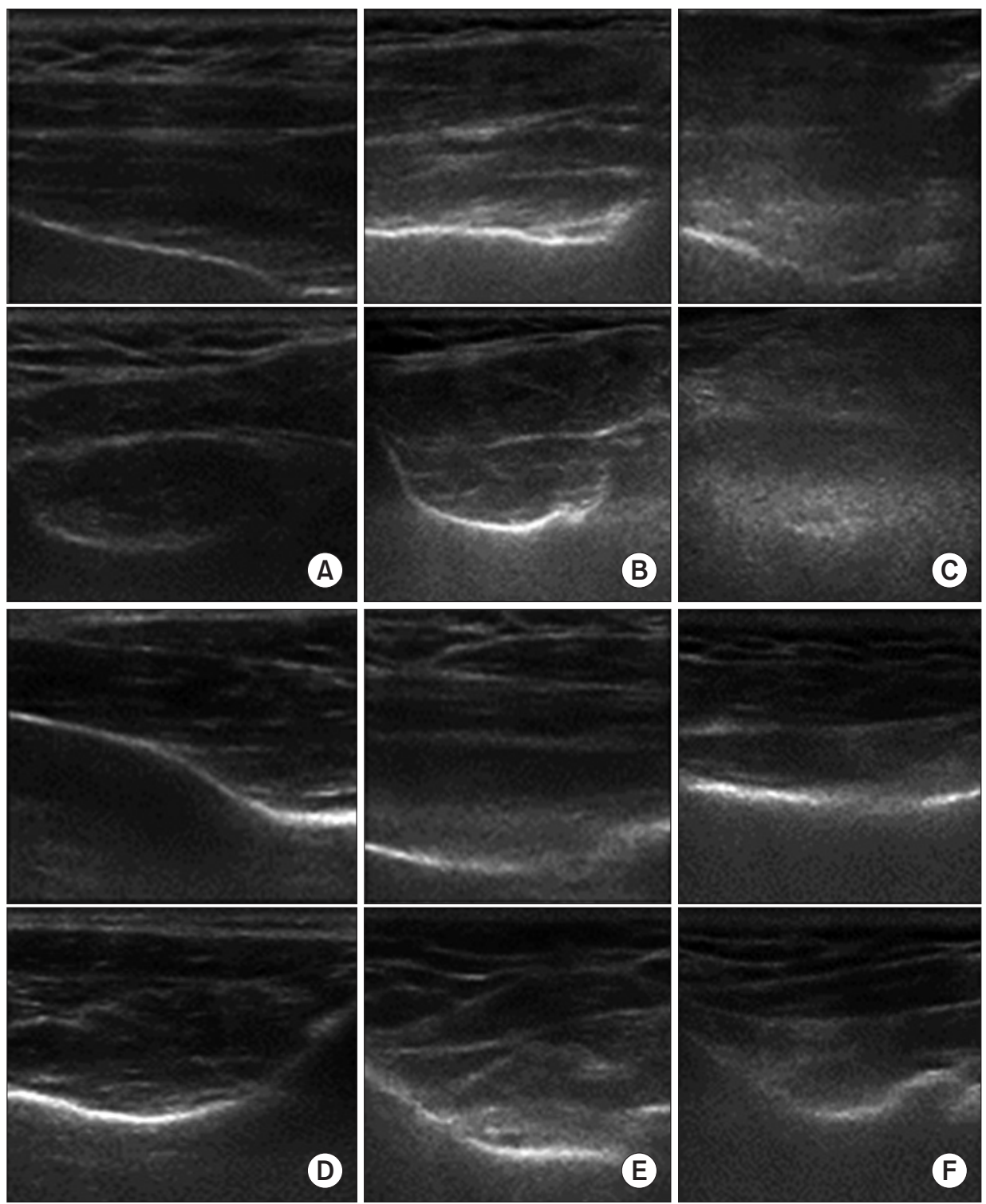

Fig. 2. Strobel's classification by ultrasonography. (A) Long- and short-axis views showing grade 0 echogenicity and grade 0 architecture of the supraspinatus muscle, which is isoechoic to the overlying muscle and has clearly visible intramuscular tendons and an identifiable pennate pattern. (B) Long- and short-axis views showing grade 1 echogenicity and grade 1 architecture of the supraspinatus muscle, which slightly increased echogenicity compared with the overlying muscle and partially visible intramuscular tendons and pennate pattern. (C) Long-and short-axis views showing grade 2 echogenicity and grade 2 architecture of the supraspinatus muscle, markedly increased echogenicity compared with the overlying muscle and no discernible intramuscular tendons or pennate pattern. (D) Long- and short-axis views showing grade 0 echogenicity and grade 0 architecture of the infraspinatus muscle, which is isoechoic to the overlying muscle and has clearly visible intramuscular tendons and an identifiable pennate pattern. (E) Long- and short-axis views showing grade 1 echogenicity and grade 1 architecture of the infraspinatus muscle, which slightly increased echogenicity compared with the overlying muscle and partially visible intramuscular tendons and pennate pattern. (F) Long- and short-axis views showing grade 2 echogenicity and grade 2 architecture of the infraspinatus muscle, markedly increased echogenicity compared with the overlying muscle and has no discernible intramuscular tendons or pennate pattern. 
Park et al. Ultrasonographic Evaluation of Rotator Cuff Muscle

Clinics in Orthopedic Surgery • Vol. 12, No. 1, $2020 \bullet$ www.ecios.org

the spinoglenoid notch was chosen for the grading. ${ }^{6,7,12,14)}$ This image was captured from each patient's MRI scan. Patient names were removed from all images, and the images were then numbered sequentially from 1 to 108 .

US was performed in a standardized manner ${ }^{15)}$ by one orthopedic surgeon (WKJ) with extensive experience. All US examinations were performed using a highfrequency linear-array transducer $(7.5-15 \mathrm{MHz})$ with an HD11 scanner (Philips, Bothell, WA, USA). The long heads of the biceps, subscapularis, supraspinatus, and infraspinatus tendons were examined by using established protocols. $^{9}$

\section{Assessment of FI in the Rotator Cuff Muscles}

The amount of FI on MRI was graded according to Fuch's modification of the Goutallier classification, as follows ${ }^{5,7}$ : grade 0 , no fatty deposits; grade 1, some fatty streaks; grade 2 , less fat than muscle; grade 3 , as much fat as muscle; and grade 4, more fat than muscle (Fig. 1).

Direct comparison with the three-point US grading scale $(0,1,2)$ was performed by collapsing the five-point MRI grading scale into a three-point scale (i.e., Goutallier grades 0 and 1 were converted to grade 0 on the threepoint scale, Goutallier grade 2 was converted to grade 1 , and Goutallier grades 3 and 4 became grade 2). To investigate the agreement on the presence of FI, the MRI classification was also collapsed into a dichotomous scale; Goutallier grades 0,1 , and 2 were converted to absent to mild on the dichotomous scale, while Goutallier grades 3 and 4 became advanced.

To assess FI on US, the echogenicity and architecture of the supraspinatus and infraspinatus were examined by the three-point scale described by Strobel et al. ${ }^{13)}$ in both long- and short-axis scans (Table 1). To investigate the supraspinatus muscle in the long axis, the transducer was placed parallel to the long axis of the muscle belly, with the spinoglenoid notch at the center of the transducer.

To investigate the supraspinatus muscle in the short axis, the transducer was placed perpendicular to the long axis of the muscle belly, with the spinoglenoid notch at the center of the transducer. The echogenicity of the supraspinatus was assessed in comparison with the echogenicity of the overlying trapezius. The architecture was assessed on the basis of the visibility of the intramuscular tendons and the normal pennate pattern (Fig. 2A-C).

To investigate the infraspinatus muscle in the long axis, the transducer was placed parallel to the long axis of the muscle belly, parallel to the central aponeurosis. To investigate the infraspinatus muscle in the short axis, the transducer was placed perpendicular to the long axis of the muscle belly, with the scapular spine at the center of the transducer. The echogenicity of the infraspinatus was assessed in comparison with that of the overlying deltoid. The architecture was assessed on the basis of the visibility of the intramuscular tendons and the normal pennate pattern (Fig. 2D-F).

The US images were saved as JPEG (Joint Photographic Experts Group) files. The images were numbered sequentially from 1 to 108 , with the patient names removed from all images. The FI status based on the saved images was graded by two orthopedic surgeons (rater 1 [WKJ] and 2 [BKP]) in a blinded fashion. The evaluation was performed twice with a 1-week interval.

Similar to the dichotomous scale for MRI findings, the classification based on US findings was collapsed into a dichotomous scale (i.e., US grades 0 and 1 were converted to absent to mild and grade 2 became advanced) to investigate the agreement on the presence of FI.

\section{Statistical Analysis}

The agreement between MRI and US for grading the degree of FI was determined. The three-point US grading of echogenicity and architecture for each muscle in each axis was compared with the MRI grading for the same muscle. The MRI grading scale was collapsed from a fivepoint scale into a three-point scale, according to previous studies. ${ }^{7,11)}$ In addition, the dichotomous US grading scale was compared with the MRI dichotomous grading scale in the same manner. The agreement between US and MRI for grading FI was assessed by using the weighted Cohen's kappa (к) coefficient. The kappa values were interpreted by using the guidelines suggested by Landis and $\operatorname{Koch}^{16)}: 0$, poor; 0 to 0.20 , slight; 0.21 to 0.40 , fair; 0.41 to 0.60 , moderate; 0.61 to 0.80 , substantial; and 0.81 to 1.0 , almost perfect agreement. Data are presented as estimates with $95 \%$ confidence intervals (CIs). Data analysis was performed using published statistical software. Furthermore, for the investigation of advanced FI in the rotator cuff muscles, the sensitivity, specificity, positive predictive value, negative predictive value, and accuracy of both modalities were calculated. Intraobserver reliabilities were assessed using the weighted Cohen's $\mathrm{k}$ coefficient. A total of 108 shoulders were evaluated twice with a 1-week interval by one orthopedic surgeon (WKJ). The status of the rotator cuff tendons and muscles was not known to the raters before the examination. 
Park et al. Ultrasonographic Evaluation of Rotator Cuff Muscle

Clinics in Orthopedic Surgery • Vol. 12, No. 1, $2020 \bullet$ www.ecios.org

\section{RESULTS}

\section{Agreement between US and MRI in the Evaluation of FI}

The comparisons between the three-point grading system on MRI and US are summarized in Tables 2-4. For the supraspinatus muscle in the long axis, the agreement between the three-point US grading of echogenicity and the MRI scales was $K=0.21$ (95\% CI, 0.05 to 0.36 ). The agreement between the three-point US grading of architecture and the MRI scales was $\mathrm{K}=0.35$ (95\% CI, 0.20 to 0.50 ). For the supraspinatus muscle in the short axis, the agreement between the three-point US grading of echogenicity and the MRI scales was $\mathrm{K}=0.26$ (95\% CI, 0.10 to 0.42 ). The agreement between the three-point US grading of architecture and the MRI scales was $\mathrm{K}=0.32$ (95\% CI, 0.16 to 0.48 ) (Table 2).

For the infraspinatus muscle in the long axis, the agreement between the three-point US grading of echogenicity and the MRI scales was $\mathrm{K}=0.34$ (95\% CI, 0.17 to
0.50). The agreement between the three-point US grading of architecture and the MRI scales was $\mathrm{K}=0.28$ (95\% CI, 0.12 to 0.44 ). For the infraspinatus muscle in the short axis, the agreement between the three-point US grading of echogenicity and the MRI scales was $\mathrm{K}=0.40$ (95\% CI, 0.24 to 0.56 ). The agreement between the three-point US grading of architecture and the MRI scales was $\mathrm{K}=0.62(95 \%$ CI, 0.47 to 0.76 ) (Table 3).

The comparisons between the MRI and US dichotomous grading scales are summarized in Tables 5-7. For the supraspinatus muscle in the long axis, the agreement between the dichotomous US grading of echogenicity and the MRI scales was $\mathrm{K}=0.48$ (95\% CI, 0.23 to 0.73 ). The agreement between the dichotomous US grading of architecture and the MRI scales was $\mathrm{K}=0.66$ (95\% CI, 0.46 to 0.87 ). For the supraspinatus muscle in the short axis, the agreement between the dichotomous US grading of echogenicity and the MRI scales was $\mathrm{K}=0.51$ (95\% CI, 0.27 to 0.75). The agreement between the dichotomous US grad-

Table 2. Classification of the Supraspinatus in the Long and Short Axes by the Three-Point Ultrasound and Three-Point MRI Grading Systems

\begin{tabular}{|c|c|c|c|c|c|}
\hline \multirow{2}{*}{ Supraspinatus } & \multirow{2}{*}{ US grade } & \multicolumn{4}{|c|}{ MRI grade } \\
\hline & & 0 (Goutallier 0,1 ) & 1 (Goutallier 2) & 2 (Goutallier 3,4 ) & Total \\
\hline \multicolumn{6}{|l|}{ Long axis } \\
\hline \multirow[t]{4}{*}{ Echogenicity } & 0 & 39 & 23 & 2 & 64 \\
\hline & 1 & 14 & 11 & 6 & 31 \\
\hline & 2 & 1 & 3 & 7 & 11 \\
\hline & Total & 54 & 37 & 15 & 106 \\
\hline \multirow[t]{4}{*}{ Architecture } & 0 & 31 & 13 & 1 & 45 \\
\hline & 1 & 21 & 21 & 3 & 45 \\
\hline & 2 & 2 & 3 & 11 & 16 \\
\hline & Total & 54 & 37 & 15 & 106 \\
\hline \multicolumn{6}{|l|}{ Short axis } \\
\hline \multirow[t]{4}{*}{ Echogenicity } & 0 & 41 & 22 & 1 & 64 \\
\hline & 1 & 12 & 11 & 6 & 29 \\
\hline & 2 & 1 & 4 & 8 & 13 \\
\hline & Total & 54 & 37 & 15 & 106 \\
\hline \multirow[t]{4}{*}{ Architecture } & 0 & 30 & 14 & 2 & 46 \\
\hline & 1 & 21 & 20 & 2 & 43 \\
\hline & 2 & 3 & 3 & 11 & 17 \\
\hline & Total & 54 & 37 & 15 & 106 \\
\hline
\end{tabular}

MRI: magnetic resonance imaging, US: ultrasonography. 
Park et al. Ultrasonographic Evaluation of Rotator Cuff Muscle

Clinics in Orthopedic Surgery • Vol. 12, No. 1, $2020 \bullet$ www.ecios.org

Table 3. Classification of the Infraspinatus in the Long and Short Axes Using the Three-Point Ultrasound and Three-Point MRI Grading Systems

\begin{tabular}{|c|c|c|c|c|c|}
\hline \multirow{2}{*}{ Infraspinatus } & \multirow{2}{*}{ US grade } & \multicolumn{4}{|c|}{ MRI grade } \\
\hline & & 0 (Goutallier 0,1$)$ & 1 (Goutallier 2) & 2 (Goutallier 3,4 ) & Total \\
\hline \multicolumn{6}{|l|}{ Long axis } \\
\hline \multirow[t]{4}{*}{ Echogenicity } & 0 & 47 & 16 & 0 & 63 \\
\hline & 1 & 15 & 16 & 3 & 34 \\
\hline & 2 & 0 & 4 & 5 & 9 \\
\hline & Total & 62 & 36 & 8 & 106 \\
\hline \multirow[t]{4}{*}{ Architecture } & 0 & 54 & 23 & 0 & 77 \\
\hline & 1 & 8 & 11 & 5 & 24 \\
\hline & 2 & 0 & 2 & 3 & 5 \\
\hline & Total & 62 & 36 & 8 & 106 \\
\hline \multicolumn{6}{|l|}{ Short axis } \\
\hline \multirow[t]{4}{*}{ Echogenicity } & 0 & 49 & 14 & 0 & 63 \\
\hline & 1 & 13 & 19 & 4 & 36 \\
\hline & 2 & 0 & 3 & 4 & 7 \\
\hline & Total & 62 & 26 & 8 & 106 \\
\hline \multirow[t]{4}{*}{ Architecture } & 0 & 54 & 10 & 0 & 64 \\
\hline & 1 & 7 & 24 & 2 & 33 \\
\hline & 2 & 1 & 2 & 6 & 9 \\
\hline & Total & 62 & 36 & 8 & 106 \\
\hline
\end{tabular}

MRI: magnetic resonance imaging, US: ultrasonography.

\section{Table 4. Agreement between MRI and US for Grading Fatty Infiltration Using the Three-Point Scales}

\begin{tabular}{ccc}
\hline \multirow{2}{*}{ Muscle } & \multicolumn{2}{c}{ Agreement-Weighted Kappa $(95 \% \mathrm{CI})$} \\
\cline { 2 - 3 } Supraspinatus & Long axis & Short axis \\
\hline Echogenicity & 0.21 & 0.26 \\
\hline Architecture & 0.35 & 0.32 \\
\hline Infraspinatus & & \\
\hline Echogenicity & 0.34 & 0.4 \\
\hline Architecture & 0.28 & 0.62 \\
\hline
\end{tabular}

MRI: magnetic resonance imaging, US: ultrasonography, Cl: confidence interval. ing of architecture and the MRI scales was $\mathrm{K}=0.63(95 \%$ CI, 0.43 to 0.84 ) (Table 5).

For the infraspinatus muscle in the long axis, the agreement between the dichotomous US grading of echogenicity and the MRI scales was $\mathrm{K}=0.55$ (95\% CI, 0.26 to 0.85 ). The agreement between the dichotomous US grading of architecture and the MRI scales was $\mathrm{K}=0.64(95 \%$ CI, 0.35 to 0.93 ). For the infraspinatus muscle in the short axis, the agreement between the dichotomous US grading of echogenicity and the MRI scales was $\mathrm{K}=0.50(95 \% \mathrm{CI}$, 0.18 to 0.82 ). The agreement between the dichotomous US grading of architecture and the MRI scales was $\mathrm{K}=0.68$ ( $95 \%$ CI, 0.42 to 0.94 ) (Table 6). The sensitivity, specificity, positive predictive value, negative predictive value, and accuracy of the detection of advanced FI were all high in 
Park et al. Ultrasonographic Evaluation of Rotator Cuff Muscle

Clinics in Orthopedic Surgery • Vol. 12, No. 1, $2020 \bullet$ www.ecios.org

Table 5. Classification of the Supraspinatus in the Long and Short Axes by the Dichotomous US and Dichotomous MRI Grading Systems

\begin{tabular}{|c|c|c|c|c|}
\hline \multirow[b]{2}{*}{ Supraspinatus } & \multirow[b]{2}{*}{ US grade } & \multicolumn{3}{|c|}{ MRI grade } \\
\hline & & $\begin{array}{c}\text { Absent to mild } \\
\text { (Goutallier } 0,1,2)\end{array}$ & $\begin{array}{c}\text { Advanced } \\
\text { (Goutallier 3, 4) }\end{array}$ & Total \\
\hline \multicolumn{5}{|l|}{ Long axis } \\
\hline \multirow[t]{3}{*}{ Echogenicity } & Absent to mild $(0,1)$ & 87 & 8 & 95 \\
\hline & Advanced (2) & 4 & 7 & 11 \\
\hline & Total & 91 & 15 & 106 \\
\hline \multirow[t]{3}{*}{ Architecture } & Absent to mild $(0,1)$ & 86 & 4 & 90 \\
\hline & Advanced (2) & 5 & 11 & 16 \\
\hline & Total & 91 & 15 & 106 \\
\hline \multicolumn{5}{|l|}{ Short axis } \\
\hline \multirow[t]{3}{*}{ Echogenicity } & Absent to mild $(0,1)$ & 86 & 7 & 93 \\
\hline & Advanced (2) & 5 & 8 & 13 \\
\hline & Total & 91 & 15 & 106 \\
\hline \multirow[t]{3}{*}{ Architecture } & Absent to mild $(0,1)$ & 85 & 4 & 89 \\
\hline & Advanced (2) & 6 & 11 & 17 \\
\hline & Total & 91 & 15 & 106 \\
\hline
\end{tabular}

US: ultrasonography, MRI: magnetic resonance imaging.

both axes (Table 8 ).

\section{Intraobserver Reliability of US}

The intraobserver reliability had a weighted $\mathrm{k}$ of $0.69(95 \%$ CI, 0.61 to 0.81 ) for the supraspinatus and $0.63(95 \% \mathrm{CI}$, 0.58 to 0.73 ) for the infraspinatus.

\section{DISCUSSION}

This study was performed to investigate the diagnostic performance and reliability of US for the evaluation of FI in the rotator cuff muscles, as compared with reference MRI. MRI has been widely accepted as a useful modality for grading FI. ${ }^{12,13)}$ However, US has several merits over MRI, including real-time evaluation, tolerance for metallic implants, and inexpensiveness.

Based on the three-point scales, the US grading in the short axis of the infraspinatus showed significant agreement with the MRI grading of the infraspinatus only, especially in terms of architecture $(\kappa=0.62)$. Using the dichotomous scales, the agreement between MRI and US improved in both axes for the supraspinatus and infraspinatus muscles. The agreement on architecture in all views was higher than the agreement on echogenicity. This may be because echogenicity can be altered by controlling the time gain compensation (TGC) during US scanning. Since the US wave is attenuated or absorbed when passing through tissue, the operator usually increases the TGC gaze in deeper areas. Thus, the echogenicity of both the supraspinatus and infraspinatus muscles was increased compared with that of the overlying trapezius and deltoid muscles.

On both the three-point and dichotomous scales, the agreement for the infraspinatus was higher than that for the supraspinatus. This might be because of the difference in the thickness and coverage amount of overlying muscles. The supraspinatus is mostly covered by the trapezius, whereas the infraspinatus is partially covered by the deltoid; therefore, there could be more interference when visualizing the supraspinatus, which is deeper than the infraspinatus.

In the previous study by Wall et al., ${ }^{4)}$ US evaluation of FI in the rotator cuff muscles was graded according to the Strobel grading scales, similar to this study. In their study, however, it was unclear how to combine and interpret the various results of the components (echogenicity 
Park et al. Ultrasonographic Evaluation of Rotator Cuff Muscle

Clinics in Orthopedic Surgery • Vol. 12, No. 1, $2020 \bullet$ www.ecios.org

Table 6. Classification of the Infraspinatus in the Long and Short Axes by the Dichotomous US and Dichotomous MRI Grading Systems

\begin{tabular}{|c|c|c|c|c|}
\hline \multirow[b]{2}{*}{ Infraspinatus } & \multirow[b]{2}{*}{ US grade } & \multicolumn{3}{|c|}{ MRI grade } \\
\hline & & $\begin{array}{c}\text { Absent to mild } \\
\text { (Goutallier } 0,1,2)\end{array}$ & $\begin{array}{c}\text { Advanced } \\
\text { (Goutallier 3, 4) }\end{array}$ & Total \\
\hline \multicolumn{5}{|l|}{ Long axis } \\
\hline \multirow[t]{3}{*}{ Echogenicity } & Absent to mild $(0,1)$ & 94 & 3 & 97 \\
\hline & Advanced (2) & 4 & 5 & 5 \\
\hline & Total & 98 & 8 & 106 \\
\hline \multirow[t]{3}{*}{ Architecture } & Absent to mild $(0,1)$ & 96 & 3 & 99 \\
\hline & Advanced (2) & 2 & 5 & 7 \\
\hline & Total & 98 & 8 & 106 \\
\hline \multicolumn{5}{|l|}{ Short axis } \\
\hline \multirow[t]{3}{*}{ Echogenicity } & Absent to mild $(0,1)$ & 95 & 4 & 99 \\
\hline & Advanced (2) & 3 & 4 & 7 \\
\hline & Total & 98 & 8 & 106 \\
\hline \multirow[t]{3}{*}{ Architecture } & Absent to mild $(0,1)$ & 95 & 2 & 97 \\
\hline & Advanced (2) & 3 & 6 & 9 \\
\hline & Total & 98 & 8 & 106 \\
\hline
\end{tabular}

US: ultrasonography, MRI: magnetic resonance imaging.

Table 7. Agreement between MRI and US for Grading Fatty Infiltration by Using Dichotomous Scales

\begin{tabular}{ccc} 
& \multicolumn{2}{c}{ Agreement-Weighted Kappa $(95 \% \mathrm{CI})$} \\
\cline { 2 - 3 } Muscle & Long axis & Short axis \\
\hline Supraspinatus & & 0.51 \\
Echogenicity & 0.48 & 0.63 \\
\hline Architecture & 0.66 & \\
Infraspinatus & & 0.50 \\
\hline Echogenicity & 0.55 & 0.68 \\
\hline Architecture & 0.64 & $\mathrm{Cl}: \mathrm{confidence}$ \\
\hline
\end{tabular}

$\mathrm{MRI}$, magnetic resonance imaging; US, ultrasonography, $\mathrm{Cl}$ : confidence interval.

and architecture) in all axes. For example, if US of the supraspinatus in the short axis showed grade I echogenicity and grade II architecture, the way in which FI was to be graded was not described. In this respect, our study refined the grading of FI by clearly classifying the interpretation of combined findings.
It is well known that atrophy and FI in the rotator cuff muscles, particularly the infraspinatus, play significant roles in determining the functional outcome after cuff repair. Therefore, given the substantial correlation, specificity, and accuracy of US, it is meaningful to confirm the FI status of the rotator cuff by using US before an operation for rotator cuff tear to facilitate an estimation of the potential degree of operative repair, especially in the short-axis view of the infraspinatus.

US has certain intrinsic limitations that should be mentioned. Evaluation of US findings depends on the experience and skills of the observer. In addition, US is difficult to perform in obese patients owing to the low penetration rate of the US wave into deep tissue. In our study, the overlying muscle, deltoid or trapezius, was used as a reference for the grading of FI via US. Aging muscles accumulate fat, ${ }^{14,17,18)}$ and it is common to see fatty streaks in the deltoid and trapezius muscles in the MRIs of elderly patients.

There are limitations in our study. First, we retrospectively evaluated FI in the rotator cuff muscles with US images saved as JPEG files, which may have led to misinterpretations compared with real-time evaluation. Never- 
Park et al. Ultrasonographic Evaluation of Rotator Cuff Muscle

Clinics in Orthopedic Surgery • Vol. 12, No. 1, $2020 \bullet$ www.ecios.org

Table 8. Sensitivity, Specificity, Positive Predictive Value, Negative Predictive Value, and Accuracy of the Grading of Fatty Infiltration in the Rotator Cuff Muscles by Using Dichotomous Scales

\begin{tabular}{|c|c|c|c|c|c|}
\hline Muscle & Sensitivity (\%) & Specificity (\%) & $\begin{array}{l}\text { Positive predictive } \\
\text { value }(\%)\end{array}$ & $\begin{array}{c}\text { Negative predictive } \\
\text { value }(\%)\end{array}$ & Accuracy $(\%)$ \\
\hline \multicolumn{6}{|c|}{ Supraspinatus (long axis) } \\
\hline Echogenicity & 47 & 96 & 64 & 92 & 89 \\
\hline Architecture & 73 & 95 & 69 & 96 & 92 \\
\hline \multicolumn{6}{|c|}{ Supraspinatus (short axis) } \\
\hline Echogenicity & 53 & 95 & 62 & 93 & 89 \\
\hline Architecture & 73 & 94 & 65 & 96 & 91 \\
\hline \multicolumn{6}{|c|}{ Infraspinatus (long axis) } \\
\hline Echogenicity & 63 & 96 & 56 & 97 & 94 \\
\hline Architecture & 63 & 98 & 71 & 97 & 95 \\
\hline \multicolumn{6}{|c|}{ Infraspinatus (short axis) } \\
\hline Echogenicity & 50 & 97 & 57 & 96 & 94 \\
\hline Architecture & 75 & 97 & 67 & 98 & 95 \\
\hline
\end{tabular}

theless, physicians sometimes need to evaluate US images taken by other operators. Therefore, the evaluation of saved images has clinical relevance. Second, the subscapularis and teres minor muscles, which are parts of the rotator cuff, were not included in this study. We analyzed US images in detail, and the data would have been more complicated if the subscapularis and teres minor muscles were included. Third, we analyzed the MRI scale. The five-point scale was compared as the three-point and dichotomous scales, based on previous reports and our clinical experience. This reduction could have introduced bias, and the effects of such a bias on reliability are unknown. However, collapsing the MRI scale was necessary to investigate clini- cal relevance.

In conclusion, US is an effective method of detecting advanced FI in the rotator cuff muscles, with good intraobserver reliabilities. In all stages of FI, the infraspinatus muscle can be assessed more accurately than the supraspinatus muscle. Furthermore, architecture is a more decisive component of FI status than echogenicity.

\section{CONFLICT OF INTEREST}

No potential conflict of interest relevant to this article was reported.

\section{REFERENCES}

1. Cho NS, Rhee YG. The factors affecting the clinical outcome and integrity of arthroscopically repaired rotator cuff tears of the shoulder. Clin Orthop Surg. 2009;1(2):96-104.

2. Goutallier D, Postel JM, Bernageau J, Lavau L, Voisin MC. Fatty muscle degeneration in cuff ruptures: pre- and postoperative evaluation by CT scan. Clin Orthop Relat Res. 1994;(304):78-83.

3. Shen PH, Lien SB, Shen HC, Lee CH, Wu SS, Lin LC. Longterm functional outcomes after repair of rotator cuff tears correlated with atrophy of the supraspinatus muscles on magnetic resonance images. J Shoulder Elbow Surg.
2008;17(1 Suppl):1S-7S.

4. Wall LB, Teefey SA, Middleton WD, et al. Diagnostic performance and reliability of ultrasonography for fatty degeneration of the rotator cuff muscles. J Bone Joint Surg Am. 2012;94(12):e83.

5. Yoo JC, Ahn JH, Yang JH, Koh KH, Choi SH, Yoon YC. Correlation of arthroscopic repairability of large to massive rotator cuff tears with preoperative magnetic resonance imaging scans. Arthroscopy. 2009;25(6):573-82.

6. Fuchs B, Weishaupt D, Zanetti M, Hodler J, Gerber C. Fatty 
degeneration of the muscles of the rotator cuff: assessment by computed tomography versus magnetic resonance imaging. J Shoulder Elbow Surg. 1999;8(6):599-605.

7. Goutallier D, Postel JM, Gleyze P, Leguilloux P, Van Driessche S. Influence of cuff muscle fatty degeneration on anatomic and functional outcomes after simple suture of fullthickness tears. J Shoulder Elbow Surg. 2003;12(6):550-4.

8. Prickett WD, Teefey SA, Galatz LM, Calfee RP, Middleton WD, Yamaguchi K. Accuracy of ultrasound imaging of the rotator cuff in shoulders that are painful postoperatively. J Bone Joint Surg Am. 2003;85(6):1084-9.

9. Teefey SA, Hasan SA, Middleton WD, Patel M, Wright RW, Yamaguchi K. Ultrasonography of the rotator cuff: a comparison of ultrasonographic and arthroscopic findings in one hundred consecutive cases. J Bone Joint Surg Am. 2000;82(4):498-504.

10. Teefey SA, Rubin DA, Middleton WD, Hildebolt CF, Leibold RA, Yamaguchi K. Detection and quantification of rotator cuff tears: comparison of ultrasonographic, magnetic resonance imaging, and arthroscopic findings in seventy-one consecutive cases. J Bone Joint Surg Am. 2004;86(4):708-16.

11. Forsberg AM, Nilsson E, Werneman J, Bergstrom J, Hultman E. Muscle composition in relation to age and sex. Clin Sci (Lond). 1991;81(2):249-56.
12. Khoury V, Cardinal E, Brassard P. Atrophy and fatty infiltration of the supraspinatus muscle: sonography versus MRI. AJR Am J Roentgenol. 2008;190(4):1105-11.

13. Strobel K, Hodler J, Meyer DC, Pfirrmann CW, Pirkl C, Zanetti M. Fatty atrophy of supraspinatus and infraspinatus muscles: accuracy of US. Radiology. 2005;237(2):584-9.

14. Tsubahara A, Chino N, Akaboshi K, Okajima Y, Takahashi $\mathrm{H}$. Age-related changes of water and fat content in muscles estimated by magnetic resonance (MR) imaging. Disabil Rehabil. 1995;17(6):298-304.

15. Zanetti M, Gerber C, Hodler J. Quantitative assessment of the muscles of the rotator cuff with magnetic resonance imaging. Invest Radiol. 1998;33(3):163-70.

16. Landis JR, Koch GG. The measurement of observer agreement for categorical data. Biometrics. 1977;33(1):159-74.

17. Rice CL, Cunningham DA, Paterson DH, Lefcoe MS. Arm and leg composition determined by computed tomography in young and elderly men. Clin Physiol. 1989;9(3):207-20.

18. Gladstone JN, Bishop JY, Lo IK, Flatow EL. Fatty infiltration and atrophy of the rotator cuff do not improve after rotator cuff repair and correlate with poor functional outcome. Am J Sports Med. 2007;35(5):719-28. 